Homology, Homotopy and Applications, vol.16(1), 2014, pp.333-339

\title{
A PURELY HOMOTOPY-THEORETIC PROOF OF THE BLAKERS-MASSEY THEOREM FOR $n$-CUBES
}

\author{
BRIAN A. MUNSON \\ (communicated by Nicholas J. Kuhn)
}

\begin{abstract}
Goodwillie's proof of the Blakers-Massey Theorem for $n$ cubes relies on a lemma whose proof invokes transversality. The rest of his proof follows from general facts about cubes of spaces and connectivities of maps. We present a purely homotopytheoretic proof of this lemma. The methods are elementary, using a generalization and modification of an argument originally due to Puppe used to prove the Blakers-Massey Theorem for squares.
\end{abstract}

\section{Introduction}

Homology has proven a useful tool because it is often computable and produces interesting invariants. In contrast, homotopy groups are usually very difficult to compute. From the standpoint of the Eilenberg-Steenrod axioms for a homology theory, the difference in the computational difficulty can be explained by the fact that homology satisfies excision while homotopy does not. However, homotopy groups satisfy excision through a range of dimensions. The most fundamental result in this direction is the Freudenthal Suspension Theorem, which gives a range of dimensions in which the homotopy groups of a highly connected space $X$ are the same as the stable homotopy groups of $X$, the latter of which satisfy excision.

Recall that a space $X$ is called $j$-connected if every map $\partial D^{i+1}=S^{i} \rightarrow X$ extends to a map $D^{i+1} \rightarrow X$ for $-1 \leqslant i \leqslant j$. A map $f: Y \rightarrow X$ is $j$-connected if for all $x \in X$, the homotopy fiber $\operatorname{hofiber}_{x}(f)=\{(y, \gamma): \gamma: I \rightarrow X, \gamma(1)=f(y), \gamma(0)=x\}$ is $(j-1)$-connected. In terms of pairs, $f: Y \rightarrow X$ is $j$-connected if $\pi_{i}(X, Y)=0$ for $1 \leqslant i \leqslant j$ and the induced map $\pi_{0} Y \rightarrow \pi_{0} X$ is surjective (here $X$ should be replaced with the mapping cylinder of $f$ so that $Y$ is a subspace).

Suppose $X$ is a $j$-connected based space. The suspension of the identity map of $X$ is adjoint to a map $X \rightarrow \Omega \Sigma X$, and the Freudenthal Suspension Theorem says that this map is $(2 j+1)$-connected. In terms of homotopy groups, the induced map $\pi_{i}(X) \rightarrow \pi_{i+1} \Sigma X$ is an isomorphism for $i \leqslant 2 j$ and onto for $i=2 j+1$. The stable homotopy groups $\pi_{i}^{S} X$ are defined as $\operatorname{colim}_{n} \pi_{i+n} \Sigma^{n} X$, so that $\pi_{i} X \rightarrow \pi_{i}^{S} X$ is an

Received September 17, 2013, revised March 23, 2014; published on May 29, 2014.

2010 Mathematics Subject Classification: 55P99.

Key words and phrases: excision, Blakers-Massey theorem, Triad connectivity theorem.

Article available at http://dx.doi.org/10.4310/HHA.2014.v16.n1.a18

The Work was prepared by a U.S. federal government employee as part his official duties, and is therefore in the public domain in the United States. 
isomorphism in the range indicated. Hence the low-dimensional homotopy groups of $X$ can be replaced by groups satisfying the excision axiom.

The Freudenthal Suspension Theorem is itself a special case of the Triad Connectivity Theorem, also known as the Blakers-Massey Theorem for squares, which says that if a space $Y$ is the union of connected subspaces $Y_{1}$ and $Y_{2}$ along their connected intersection $Y_{\emptyset}=Y_{1} \cap Y_{2}$, and if $\pi_{i}\left(Y_{1}, Y_{\emptyset}\right)=0$ for $1 \leqslant i \leqslant j$ and $\pi_{i}\left(Y_{2}, Y_{\emptyset}\right)=0$ for $1 \leqslant i \leqslant l$, then the excision map $\pi_{i}\left(Y_{1}, Y_{\emptyset}\right) \rightarrow \pi_{i}\left(Y, Y_{2}\right)$ is an isomorphism for $1 \leqslant i \leqslant j+l-1$ and onto for $i=j+l$ (to obtain the Freudenthal Suspension Theorem let $Y_{\emptyset}=X$ be $j$-connected and $Y_{1}, Y_{2}$ be copies of the cone on $Y_{\emptyset}$ ). We say "for squares" because the theorem can be neatly and more symmetrically described by organizing the spaces into the square diagram

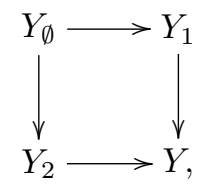

and the result can be interpreted as a range of dimensions in which either of the maps of pairs $\left(Y_{1}, Y_{\emptyset}\right) \rightarrow\left(Y, Y_{2}\right)$ or $\left(Y_{2}, Y_{\emptyset}\right) \rightarrow\left(Y, Y_{1}\right)$ induces isomorphisms in homotopy (i.e., a range in which these groups satisfy excision). Another more symmetric way to say this is that the map $Y_{\emptyset} \rightarrow \operatorname{holim}\left(Y_{1} \rightarrow Y \leftarrow Y_{2}\right)$ is $(j+l-1)$-conneced. This has generalizations to higher-order excision; for example, where $Y$ is the union of many spaces $Y_{i}$ along a common subspace $Y_{\emptyset}$.

The Blakers-Massey Theorem for $k$-cubes, also known as the $(k+1)$-ad Connectivity Theorem, is a result giving a range of dimensions in which higher-order excision holds for homotopy groups. A $k$-cube of topological spaces is a functor $\mathcal{X}=T \mapsto X_{T}$ from the poset of subsets of $\{1, \ldots, k\}$ to the category of topological spaces. Thus a 2 -cube $\mathcal{X}$ is a square

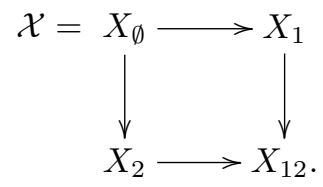

We say such a square is $j$-cocartesian if the canonical map hocolim $\left(X_{2} \leftarrow X_{\emptyset} \rightarrow\right.$ $\left.X_{1}\right) \rightarrow X_{12}$ is $j$-connected. When $j=\infty$, we say the square is homotopy cocartesian. A $k$-cube $\mathcal{X}=T \mapsto X_{T}$ is called strongly cocartesian if all its square faces are homotopy cocartesian. We say $\mathcal{X}$ is $j$-cartesian if the canonical map $X_{\emptyset} \rightarrow \operatorname{holim}_{\emptyset \neq T \subset\{1, \ldots, k\}} X_{T}$ is $j$-connected. See Section 1 and Definitions 1.3, 1.4, and 2.1 of [6] for terminology, and Section 2 for more on higher-order excision, including the results discussed in the current work.

Theorem 1.1 (Ellis-Steiner [5]). If $\mathcal{X}=T \mapsto X_{T}$ is a strongly cocartesian $k$-cube and the maps $X_{\emptyset} \rightarrow X_{\{i\}}$ are $j_{i}$-connected for all $i \in\{1, \ldots, k\}$, then $\mathcal{X}$ is $\left(1-k+\sum_{i=1}^{k} j_{i}\right)$-cartesian.

The proof of the Blakers-Massey Theorem for $k$-cubes is originally due to Barratt and Whitehead [1], with the additional hypothesis that $j_{i} \geqslant 2$ for all $i$, and was later improved as above by Ellis and Steiner [5]. In addition, Ellis and Steiner were 
able to compute the first non-trivial group of such cubes. Their techniques use cat ${ }^{k}-$ groups, following Brown and Loday $[\mathbf{2}, \mathbf{3}, \mathbf{4}]$, and as a result the proofs require extra machinery and are algebraic in nature. At the expense of losing information about the first non-trivial group, one can use the simpler and more direct space-level proof due to Goodwillie [6], who was also able to prove a generalization of Theorem 1.1 to a wider class of cubes (Theorem 2.5 of $[\mathbf{6}]$ ). As Goodwillie notes, a good deal of his proof of Theorem 1.1 is quite formal, relying on general results about cubical diagrams and connectivities of maps. However, it relies on a lemma, stated below, which uses a transversality (dimension counting) argument, and as such depends on arguments from the smooth category. Our work aims to prove the lemma using only elementary homotopy theory, and alongside Goodwillie's formal arguments stands as a purely homotopy-theoretic and space-level proof of Theorem 1.1. As with proofs of excision for homology, our techniques utilize subdivision (see for instance Lemma 2.3 below).

For a positive integer $k$, let $\underline{k}=\{1, \ldots, k\}$. Let $\mathcal{X}=T \mapsto X_{T}$ be a pushout cube of spaces, formed by attaching cells $e_{j}$ of dimension $d_{j}+1$ for $1 \leqslant j \leqslant k$ to a space $X_{\emptyset}$. That is, $X_{T}=X_{\emptyset} \cup\left\{e_{j}: j \in T\right\}$ for $T \subset \underline{k}$ for some choice of attaching maps $\partial e_{j} \rightarrow X_{\emptyset}, 1 \leqslant j \leqslant k$.

Lemma 1.2. [Lemma 2.7 of [6]] With $\mathcal{X}$ as above, choose a basepoint $x \in X_{\{k\}}$, and for $T \subset \underline{k-1}$ let $\mathcal{F}(T)=$ hofiber $_{x}\left(X_{T} \rightarrow X_{T \cup\{k\}}\right)$. Then the $(k-1)$-cube $\mathcal{F}$ is

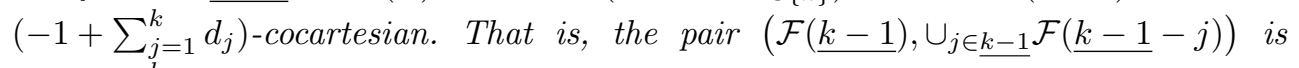
$\left(-1+\sum_{j=1}^{k} d_{j}\right)$-connected.

We learned an elementary proof of the Triad Connectivity Theorem (Theorem 1.1 in the case of squares) from tom Dieck's book [8], who credits Puppe [7]. The main theme of this proof is subdivision, much like proofs of excision for homology. We adapt these ideas to prove Lemma 1.2 without use of transversality arguments. Our proof mirrors Goodwillie's, and we replace his "dimension counting" argument with a "coordinate counting" one.

\section{Preliminaries}

We first make a simplification in the hypotheses of Lemma 1.2. If $d_{j}=-1$ for all $j$, then the conclusion of Lemma 1.2 is vacuously true. Without loss of generality $d_{k} \geqslant 0$. The basepoint $x \in X_{\{k\}}$ can be joined by a path to some point in $X_{\emptyset}$, so we may as well assume the basepoint lies in $X_{\emptyset}$ by the homotopy invariance of homotopy fibers over path components. If $d_{i}=-1$ for any other value of $i$, then $X_{\emptyset} \rightarrow X_{\{i\}}$ is the inclusion of $X_{\emptyset}$ to itself with a disjoint point added. This point plays no role in any of the homotopy fibers appearing in the cube $\mathcal{F}$, and we may ignore it altogether. More precisely, for this value of $i$ and a basepoint $x \in X_{\emptyset}$, we have $\operatorname{hofiber}_{x}\left(X_{T} \rightarrow\right.$ $\left.X_{T \cup\{k\}}\right)=\operatorname{hofiber}_{x}\left(X_{T \backslash\{i\}} \rightarrow X_{T \backslash\{i\} \cup\{k\}}\right)$ for all $T \subset \underline{k-1}$. Thus we may assume $d_{j} \geqslant 0$ for all $1 \leqslant j \leqslant k$. The remainder of this section is an adaptation of material from Section 6.9 of $[\mathbf{8}]$.

Definition 2.1. A cube $W$ in $\mathbb{R}^{n}$ is a set of the form

$$
W=W(a, \delta, L)=\left\{x \in \mathbb{R}^{n}: a_{i} \leqslant x_{i} \leqslant a_{i}+\delta \text { for } i \in L, x_{i}=a_{i} \text { for } i \notin L\right\},
$$

where $a=\left(a_{1}, \ldots, a_{n}\right) \in \mathbb{R}^{n}, \delta>0$, and $L \subset\{1, \ldots, n\}$ (possibly empty). Define 
$\operatorname{dim}(W)=|L|$. The boundary $\partial W$ of $W$ is the set of all $x$ in $W$ such that $x_{i}=a_{i}$ or $x_{i}=a_{i}+\delta$ for at least one value of $i \in L$. The boundary $\partial W$ is a union of faces. A face of a cube is also a cube.

Definition 2.2. With $W$ as above, for each $j=1$ to $k$ define

$$
K_{p}^{j, k}(W)=\left\{x \in W: \frac{\delta(j-1)}{k}+a_{i}<x_{i}<\frac{\delta j}{k}+a_{i} \text { for at least } p \text { values of } i \in L\right\} .
$$

If $p \leqslant q$, then $K_{q}^{j, k}(W) \subset K_{p}^{j, k}(W)$. The following lemma gives the basic technical deformation result, with statement and proof a straightforward generalization of 6.9.1 of $[8]$.

Lemma 2.3. Let $Y$ be a space with a subspace $A \subset Y, W$ a cube, $j, k$ positive integers with $j \leqslant k$, and $f: W \rightarrow Y$ a map. Suppose that for $p \leqslant \operatorname{dim}(W)$ we have

$$
f^{-1}(A) \cap W^{\prime} \subset K_{p}^{j, k}\left(W^{\prime}\right)
$$

for all cubes $W^{\prime} \subset \partial W$. Then there exists a map $g: W \rightarrow Y$ homotopic to $f$ relative to $\partial W$ such that

$$
g^{-1}(A) \subset K_{p}^{j, k}(W) .
$$

Proof. Without loss of generality $W=I^{n}, n \geqslant 1$. We will construct a map $h: I^{n} \rightarrow I^{n}$ homotopic to the identity and define $g$ to be the composition of $f$ with $h$. Let $x=\left(\frac{2 j-1}{2 k}, \ldots, \frac{2 j-1}{2 k}\right)$ be the center of the cube $\left[\frac{j-1}{k}, \frac{j}{k}\right]^{n}$. For a ray $y$ emanating from $x$, let $P(y)$ be its intersection with $\partial\left[\frac{j-1}{k}, \frac{j}{k}\right]^{n}$ and $Q(y)$ its intersection with $\partial I^{n}$. Let $h$ map the segment from $P(y)$ to $Q(y)$ onto the point $Q(y)$ and the segment from $x$ to $P(y)$ affinely onto the segment from $x$ to $Q(y)$. Clearly $h$ is homotopic to the identity of $I^{n}$ relative to $\partial I^{n}$, and so $g=f \circ h$ is homotopic to $f$ relative to $\partial I^{n}$. It remains to check that $g$ satisfies the property in the conclusion of the lemma.

Suppose $z \in I^{n}$ and $g(z) \in A$. Write $z=\left(z_{1}, \ldots, z_{n}\right)$. If $z \in\left(\frac{j-1}{k}, \frac{j}{k}\right)^{n}$, then $z \in K_{n}^{j, k}(W) \subset K_{p}^{j, k}(W)$ and we are done. Now assume there exists $i$ so that either $z_{i} \geqslant \frac{j}{k}$ or $z_{i} \leqslant \frac{j-1}{k}$. Then by definition of $h$, we have $h(z) \in \partial I^{n}$, so $h(z) \in W^{\prime}$ for some face $W^{\prime}$ of dimension $n-1$. Since $g(z)=f(h(z)) \in A, h(z) \in f^{-1}(A)$, then by assumption $h(z) \in K_{p}^{j, k}\left(W^{\prime}\right)$. Thus for at least $p$ values of $i$, we have $\frac{j-1}{k}<h(z)_{i}<\frac{j}{k}$, where $h(z)_{i}$ denotes the $i$ th coordinate of $h(z)$. By definition of $h$,

$$
h(z)_{i}=\frac{2 j-1}{2 k}+t\left(z_{i}-\frac{2 j-1}{2 k}\right) \quad \text { for } t \geqslant 1 .
$$

Inserting this expression into the previous inequalities and solving for $z_{i}$ yields

$$
-\frac{1}{t 2 k}+\frac{2 j-1}{2 k}<z_{i}<\frac{1}{t 2 k}+\frac{2 j-1}{2 k} .
$$

Since the lower bound increases with $t$ and the upper bound decreases with $t$, substituting $t=1$ into each gives

$$
\frac{j-1}{k}<z_{i}<\frac{j}{k}
$$

so that $z \in K_{p}^{j, k}(W)$. 
Suppose $Y$ is a space with open subsets $Y_{\emptyset}, Y_{1}, \ldots, Y_{k}$ such that $Y$ is the union of $Y_{1}, \ldots, Y_{k}$ along $Y_{\emptyset}$. Let $f: I^{n} \rightarrow Y$ be a map. By the Lebesgue Covering Lemma, we can decompose $I^{n}$ into cubes $W$ such that $f(W) \subset Y_{j}$ for some $j$ depending on $W$. The following is a generalization of Theorem 6.9 .2 in $[\mathbf{8}]$, as is its proof.

Theorem 2.4. With the $Y_{j}$ and $f$ as above, assume that for each $j,\left(Y_{j}, Y_{\emptyset}\right)$ is $d_{j}$-connected, with $d_{j} \geqslant 0$ (i.e., $Y_{\emptyset} \rightarrow Y_{j}$ is $d_{j}$-connected). Then there is a homotopy $f_{t}$ of $f$ such that

1. If $f(W) \subset Y_{j}$, then $f_{t}(W) \subset Y_{j}$ for all $t$,

2. If $f(W) \subset Y_{\emptyset}$, then $f_{t}(W)=f(W)$ for all $t$, and

3. If $f(W) \subset Y_{j}$, then $f_{1}^{-1}\left(Y_{j} \backslash Y_{\emptyset}\right) \cap W \subset K_{d_{j}+1}^{j, k}(W)$.

Proof. Let $C^{l}$ be the union of cubes $W$ with $\operatorname{dim}(W) \leqslant l$. The homotopy $f_{t}$ is constructed inductively over $C^{k} \times I$. If $\operatorname{dim}(W)=0$, then if $f(W) \subset Y_{\emptyset}$, we simply let $f_{t}=f$, which achieves the second condition. Note that if $f(W) \subset Y_{j} \cap Y_{i}$ for $i \neq j$, then $f(W) \subset Y_{\emptyset}$. Hence we only need consider what happens if $f(W) \subset Y_{j}$ and $f(W) \not \subset Y_{i}$ for all $i \neq j$. In this case, if $f(W) \subset Y_{j}$ and $f(W) \not \subset Y_{i}$ for all $i \neq j$, then since $\left(Y_{j}, Y_{\emptyset}\right)$ is $d_{j}$-connected and $d_{j} \geqslant 0$, choose a path from $f(W)$ to some point in $Y_{\emptyset}$ and use this as the homotopy, so that $f_{1}(W) \subset Y_{\emptyset}$. Then clearly the first condition holds and so does the third (in this case, the third condition says the empty set is contained in $K_{d_{i+1}}^{j, k}(W)$ ). This proves the base case.

Since the inclusion $\partial W \subset W$ is a cofibration for any cube $W$, we may extend this homotopy over all cubes $W$ so that the first and second conditions hold. By induction suppose that $f$ has been changed by a homotopy satisfying all three conditions for cubes of dimension less than $l$, and let $W$ be a cube with $\operatorname{dim}(W)=l$. If $f(W) \subset Y_{\emptyset}$, we let $f_{t}=f$ as usual. If $f(W) \subset Y_{j}$ and $f(W) \not \subset Y_{i}$ for all $i \neq j$, then:

- If $\operatorname{dim}(W)=l \leqslant d_{j}$, then since $\left(Y_{j}, Y_{\emptyset}\right)$ is $d_{j}$-connected there is a homotopy $f_{t}$ of $f$ relative to $\partial W$ such that $f_{1}(W) \subset Y_{\emptyset}$, and clearly the first and third conditions hold.

- If $\operatorname{dim}(W)=l>d_{j}$, we employ Lemma 2.3. Let $A=Y_{j} \backslash Y_{\emptyset} \subset Y_{j}$. By induction we have that, for all $W^{\prime} \subset \partial W$,

$$
f^{-1}\left(Y_{j} \backslash Y_{\emptyset}\right) \cap W^{\prime} \subset K_{l}^{j, k}\left(W^{\prime}\right) \subset K_{d_{j}+1}^{j, k}\left(W^{\prime}\right),
$$

and by Lemma 2.3 , there is a homotopy $f_{t}$ of $f$ relative to $\partial W$ such that $f_{1}^{-1}\left(Y_{j} \backslash Y_{\emptyset}\right) \cap W \subset K_{d_{j}+1}^{j, k}(W)$.

\section{3. $\quad$ Proof of Lemma 1.2}

We need to convert the strongly cocartesian cube $\mathcal{X}$ in the statement of Lemma 1.2 into one where the maps are inclusions of open sets in order to apply the previous results. For each $1 \leqslant j \leqslant k$ and corresponding cell $e_{j}$ with attaching map $f_{j}: \partial e_{j} \rightarrow X_{\emptyset}$, assume $e_{j}=D^{d_{j}+1}$, put $N_{j}=D^{d_{j}+1}-\{0\}$, and let $V_{j}$ be the interior of $D^{d_{j}+1}$. Define a $k$-cube $\mathcal{Y}=S \mapsto Y_{S}$ for $S \subset \underline{k}$ as follows. Let $U=X_{\emptyset} \cup_{j=1}^{n} N_{j}$. The inclusion $X_{\emptyset} \rightarrow U$ is a homotopy equivalence, and $U$ is open in $X_{\underline{k}}$. For $S \subset \underline{k}$, let $Y_{S}=U \cup_{j \in S} V_{j}$. Then $Y_{S}$ is open in $Y_{\underline{k}}=X_{\underline{k}}$ for each $S$, and the maps $Y_{S} \rightarrow Y_{T}$ for $S \subset T$ are the evident inclusions. The inclusion $X_{S} \rightarrow Y_{S}$ gives rise to a map 
of $k$-cubes $\mathcal{X} \rightarrow \mathcal{Y}$, which is an equivalence for each $S$. Now we are ready to prove Lemma 1.2.

Proof of Lemma 1.2. With $\mathcal{Y}=T \mapsto Y_{T}$ as above, choose a basepoint $y \in Y_{\emptyset}$, put $\mathcal{F}^{\prime}(T)=\operatorname{hofiber}_{y}\left(Y_{T} \rightarrow Y_{T \cup\{k\}}\right)$ for $T \subset \underline{k-1}$, and let $C$ be the contractible space $C=\operatorname{hofiber}_{y}\left(Y_{k-1} \rightarrow Y_{k-1}\right)$. As indicated in the paragraph preceding this proof, $\mathcal{F}^{\prime}(T) \simeq \mathcal{F}(T)$. Following Goodwillie's proof of Lemma 1.2 and using 1.16(a) in [6], it is enough to show that the cube $T \mapsto \mathcal{F}^{*}(T)=\mathcal{F}^{\prime}(T) \cup C$ is $\left(-1+\sum_{j} d_{j}\right)$-cocartesian; that is, that the pair

$$
(A, B)=\left(\mathcal{F}^{*}(\underline{k-1}), \cup_{j \in \underline{k-1}} \mathcal{F}^{*}(\underline{k-1}-j)\right)
$$

is $\left(-1+\sum_{j} d_{j}\right)$-connected. Note that the conclusion is automatic if $d_{j}=0$ for all $j$, since any pair $(A, B)$ is $(-1)$-connected. Let $\phi:\left(I^{n}, \partial I^{n}\right) \rightarrow(A, B)$ be a map. The map $\phi$ is adjoint to a map $\Phi: I^{n} \times I \rightarrow Y_{k}$ with boundary conditions

(B0) $\Phi(z, 0)=y \in Y_{\emptyset}$ is the basepoint for all $z \in I^{n}$,

(B1) $\Phi(z, 1) \in \cup_{j \in k-1} Y_{j}=Y_{\underline{k-1}}$ for all $z \in I^{n}$, and

(B2) for each $z \in \overline{\partial I^{n}}$ there exists $i(z) \in \underline{k}$ so that $\Phi(z, t) \in \cup_{j \neq i(z)} Y_{j}$ for all $t \in I$.

We will make a homotopy of $\Phi$ preserving (B0)-(B2) such that the last condition holds for each $z \in I^{n}$. To do this we apply Theorem 2.4 to $\Phi: I^{n} \times I \rightarrow Y_{\underline{k}}$ and obtain a decomposition of $I^{n} \times I$ into cubes $W$ such that for each $W$ there is some $j$ so that $\Phi(W) \subset Y_{j}$, and a homotopy $\Phi_{r}$ for $0 \leqslant r \leqslant 1$ of $\Phi=\Phi_{0}$ such that

1. $\Phi(W) \subset Y_{j}$ implies $\Phi_{r}(W) \subset Y_{j}$ for all $r$,

2. $\Phi(W) \subset Y_{\emptyset}$ implies $\Phi_{r}(W)=\Phi(W)$ for all $r$, and

3. $\Phi(W) \subset Y_{j}$ implies $\Phi_{1}^{-1}\left(Y_{j} \backslash Y_{\emptyset}\right) \cap W \subset K_{d_{j}+1}^{j, k}(W)$.

First we prove that $\Phi_{r}$ satisfies (B0)-(B2) for all $r$.

(B0) Since $\Phi(z, 0)=y \in Y_{\emptyset}$ is the basepoint for all $z \in I^{n}$, we have for all cubes $W \subset I^{n} \times\{0\}$ that $\Phi(W)=y$, and (2) above implies $\Phi_{r}(W)=\Phi(W)$ for all $r$, so that $\Phi_{r}(z, 0)=y$ for all $r$.

(B1) Since $\Phi(z, 1) \in \cup_{j \in k-1} Y_{j}=Y_{k-1}$ for all $z \in I^{n}$, then for all cubes $W \subset I^{n} \times\{1\}$, $\Phi(W) \subset Y_{j}$ for some $1 \leqslant j \leqslant \overline{k-1}$. Hence $\Phi_{r}(W) \subset Y_{j}$ as well by (1) above, and thus $\Phi_{r}(z, 1) \subset Y_{\underline{k-1}}$ for all $r, z$.

(B2) We know that for each $z \in \partial I^{n}$ there exists $i(z) \in \underline{k}$ so that $\Phi(\{z\} \times I) \subset$ $\cup_{j \neq i(z)} Y_{j}$. Let $W_{1}, \ldots, W_{h}$ be cubes such that $\{z\} \times I \subset W_{1} \cup \cdots \cup W_{h}$ and so that each $W_{a}$ contains a point of the form $(z, t)$ for some $t$. Since $\Phi(\{z\} \times I) \subset$ $\cup_{j \neq i(z)} Y_{j}$, for each $a=1$ to $h$ we must have $\Phi\left(W_{a}\right) \subset Y_{j(a)}$ for some $j(a) \neq i(z)$. This implies $\Phi_{r}\left(W_{1} \cup \cdots \cup W_{h}\right) \subset Y_{j(1)} \cup \cdots \cup Y_{j(h)} \subset \cup_{j \neq i(z)} Y_{j}$ for all $r$, again by (1) above.

Now we show that $\Phi_{1}$ actually satisfies the stronger condition that for each $z \in I^{n}$ there exists $i(z) \in \underline{k}$ so that $\Phi_{1}(z, t) \in \cup_{j \neq i(z)} Y_{j}$ for all $t \in I$ when $n<\sum_{j} d_{j}$. Let $\pi: I^{n} \times I \rightarrow I^{n}$ be the projection. We claim that

$$
\bigcap_{j=1}^{k} \pi\left(\Phi_{1}^{-1}\left(Y_{j} \backslash Y_{\emptyset}\right)\right)=\emptyset
$$

if $n<\sum_{j} d_{j}$. Let $y \in \pi\left(\Phi_{1}^{-1}\left(Y_{j} \backslash Y_{\emptyset}\right)\right)$ for all $j$, so that $y$ is an element of this 
intersection. For each $j$, we may choose $t_{j}$ so that $\left(y, t_{j}\right) \in \Phi^{1-}\left(Y_{j} \backslash Y_{\emptyset}\right)$, so that $y=\pi\left(y, t_{j}\right)$ and $w(j)=\left(y, t_{j}\right) \in W_{j}$ for some cube $W_{j} \subset I^{n} \times I$. Thus, for each $j$, $w(j) \in W_{j} \cap \Phi_{1}^{-1}\left(Y_{j} \backslash Y_{\emptyset}\right) \subset K_{d_{j}+1}^{j, k}$ by (3) of Theorem 2.4. This means $w(j)$ has at least $d_{j}+1$ coordinates $w(j)_{i}$ such that $a_{i}+\frac{\delta(j-1)}{k}<w(j)_{i}<a_{i}+\frac{\delta j}{k}$, where $W_{j}=$ $W(a, \delta, L)$ and $a=\left(a_{1}, \ldots, a_{n+1}\right)$. This implies that $y$ has at least $d_{j}$ coordinates $y_{i}$ satisfying the same bounds (only here the index $i$ ranges between 1 and $n$ ). For each $j$, the projection $\pi\left(W_{j}\right)$ is a cube containing $y$, and subdividing further if necessary (Theorem 2.4 clearly still applies to any such further subdivision), we may assume $\pi\left(W_{j}\right)=W$ for all $j$. Thus $y$ has at least $d_{j}$ coordinates $y_{i}$ satisfying $a_{i}+\frac{\delta(j-1)}{k}<y_{i}<a_{i}+\frac{\delta j}{k}$ for all $j$, which is impossible if $n<\sum_{j} d_{j}$, so that the intersection in Equation (1) is indeed empty. Hence there is some $i(y) \in \underline{k}$ so that $y \notin \pi\left(\Phi_{1}^{-1}\left(Y_{i(y)} \backslash Y_{\emptyset}\right)\right)$. That is, for all $t,(y, t) \notin \Phi_{1}^{-1}\left(Y_{i(y)} \backslash Y_{\emptyset}\right)$, as required.

When $n=0$, to show $\pi_{0}(B) \rightarrow \pi_{0}(A)$ is surjective our argument above requires $d_{j} \geqslant 1$ for at least one $j$. We have already noted near the beginning of the proof that the conclusion of the theorem was trivially true when $d_{j}=0$ for all $j$.

\section{Acknowledgments}

The author would like to thank the referee for a careful reading and thoughtful comments that improved the exposition of this paper, and for pointing out a gap in the proof of Lemma 1.2. This work was partially supported by ONR grants N0001412WX30191 and N0001413WX20992.

\section{References}

[1] M.G. Barratt and J.H.C. Whitehead, The first non-vanishing group of an $(n+1)$-ad, Proceedings of the London Math. Soc. 6 (1956), 417-439.

[2] R. Brown and J.-L. Loday, Excision homotopique en basse dimension, C.R. Acad. Sci. Paris Sér I Math. 298 (1984), 353-356.

[3] R. Brown and J.-L. Loday, Homotopical excision and hurewicz theorems for $n$ cubes of spaces, Proc. London Math. Soc. 54 (1987), no. 3, 176-192.

[4] R. Brown and J.-L. Loday, Van kampen theorems for diagrams of spaces, Topology 26 (1987), no. 3, 311-335.

[5] G.J. Ellis and R. Steiner, Higher-dimensional crossed modules and the homotopy groups of $(n+1)$-ads, J. Pure Appl. Algebra 46 (1987), 117-136.

[6] T.G. Goodwillie, Calculus II: Analytic functors, K-Theory 5 (1991/92), no. 4, 295-332.

[7] K.H. Kamps, T. tom Dieck and D. Puppe, Homotopietheorie, Lecture Notes in Math., no. 157, Springer, 1970.

[8] T. tom Dieck, Algebraic topology, EMS Textbooks in Mathematics, European Mathematical Society, 2008.

Brian A. Munson munson@usna. edu

Department of Mathematics, U.S. Naval Academy, 572C Holloway Road, Chauvenet Hall, Annapolis, MD 21402, USA 